\title{
Pseudoaxonal Guillain-Barré syndrome: severe demyelination mimicking axonopathy. A case with pupillary involvement
}

\author{
G N Fuller, J M Jacobs, P D Lewis, R J M Lane
}

\begin{abstract}
A patient with fulminant Guillain-Barré syndrome developed pupillary denervation, loss of all brain stem reflexes and a complete flaccid quadriparesis. Pathological studies confirmed this was due to a primarily demyelinating process and not an axonal form of Guillain-Barré syndrome.
\end{abstract}

(F Neurol Neurosurg Psychiatry 1992;55:1079-1083)

Neurophysiological and pathological studies in Guillain-Barré syndrome (GBS) have indicated that the underlying pathological process is predominantly demyelination with a lesser degree of axonal loss. ${ }^{13}$ In 1986, however, Feasby et $a l^{4}$ reported five patients with electrically inexcitable nerves, with axonal loss at necropsy in one, and suggested these cases may constitute a separate clinicopathological entity, an acute axonal form of Guillain-Barré syndrome.

We describe the clinical and pathological features of a patient with electrically inexcitable nerves who developed pupillary denervation as part of fulminant and ultimately fatal GBS and in whom the pathological process proved to be severe demyelination

\section{Case report}

A 63 year old previously healthy homosexual man noted pains in both legs and across his shoulders. Next morning he developed a generalised progressive weakness preventing him from walking. His voice became hoarse and he noted tingling on the left side of his forehead. On examination he was apyrexial, pulse $68, \mathrm{BP}$ $150 / 90$ and general examination was normal. His left pupil was noted to be larger than his right with normal reaction to light and accommodation. He had a flaccid areflexic quadriparesis with grade $3 / 5$ weakness of both arms and legs.

No sensory abnormalities were found. Over the next 12 hours his weakness progressed and he required mechanical ventilation. Investigations revealed an acellular CSF, with elevated protein $(0.66 \mathrm{~g} / \mathrm{l})$, CSF glucose $3.6 \mathrm{mmol} / 1$ (blood glucose $5.9 \mathrm{mmol} / \mathrm{l}$ ). Full blood count, ESR, urea and electrolytes, liver function tests, blood lead and thallium, urinary porphyrins, HIV antibody, HIV p24 antigen, Hepatitis B
sAg, VDRL/TPHA and CT brain scan were all normal or negative. Two days after admission he had $4 \mathrm{~mm}$ pupils unreactive to light, no eye movements on doll's eye manoeuvre or caloric testing and absent corneal reflexes. He had no cephalic or peripheral response to pain and was areflexic.

Electrophysiological studies performed three days later found absent median, ulnar and sural sensory action potentials and no motor response was obtained from stimulation of median, ulnar, common peroneal or posterior tibial nerves. EMG revealed no spontaneous activity but showed an increase in insertional activity. A sural nerve biopsy was performed six days after the onset of neuropathic symptoms. EEG showed symmetrical generalised low voltage rhythmical alpha activity of $7-11 \mathrm{~Hz}$ with mixed activity at $1 \cdot 5-6 \cdot 5 \mathrm{~Hz}$. Episodes of low frequency delta waves of about $1 \mathrm{~Hz}$ up to $50 \mathrm{uV}$ in amplitude were evident in the posterior central areas. Beta activity at $14-20 \mathrm{~Hz}$ was diffuse. No response was seen to painful or visual stimuli. Visual evoked potentials to flash and brain stem auditory evoked potentials were normal. Repeat CSF one week after admission was acellular with a protein of $1.26 \mathrm{~g} / \mathrm{l}$. Instillation of one in 1000 adrenalin to both eyes produced a $1 \mathrm{~mm}$ dilatation after 20 minutes. $0 \cdot 125 \%$ pilocarpine drops produced constriction of $1.5 \mathrm{~mm}$ at 20 minutes; instillation of $4 \%$ cocaine produced no response. Subcutaneous injection of one in 1000 histamine produced no weal at $30 \mathrm{~min}$ utes, when the weal in a normal control subject was maximal.

At this point his blood pressure began to fluctuate between $110 / 60$ and $180 / 110$ and later that day he developed sustained hypertension which was treated with nifedepine. He had plasma exchange (total discard $12 \mathrm{l}$ ) and was treated with methylprednisolone $500 \mathrm{mg}$ for five days. One week later he was able to shake his head on command and was therefore able to communicate. He continued to make progress but six days later, 18 days after his first neuropathic symptoms, he developed an irreverisble cardiac dysrhythmia and fatal cardiac arrest.

\section{Pathology}

The sural nerve histology and morphometry was performed using previously described techniques. ${ }^{5}$ Necropsy was performed using standard techniques. Brain, cranial nerves, upper cervical cord with associated dorsal and 


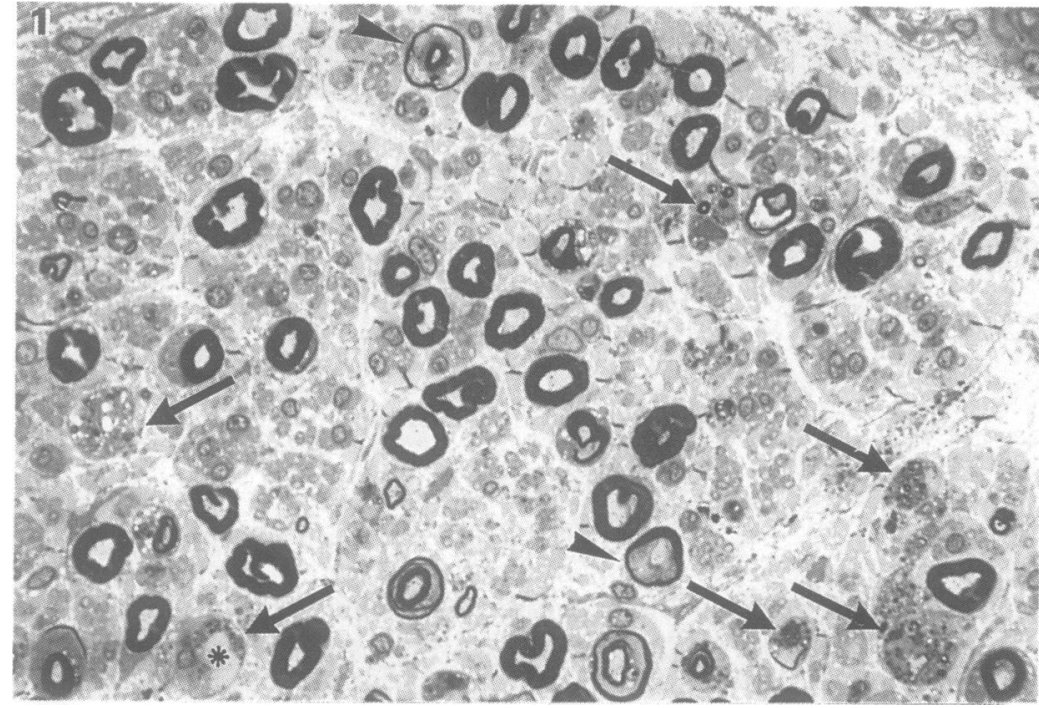

Figure 1 Transverse $1 \mu \mathrm{m}$ araldite section showing a reduced density of myelinated fibres: most of those remaining are large. Arrows mark fibres containing granular myelin debris: in only one fibre is demyelination clearly identifiable (bottom left: axon marked by asterisk). Fibres marked with arrowheads were confirmed by electron microscopy to have vesicular myelin. $\times 500$.
Figure 3 Teased fibres showing abnormalities in whole internodes adjacent to normal internodes. Arrows indicate nodes of Ranvier. Abnormal internodes are marked by arrowheads. (a) The central internode shows pale and irregular staining of its myelin sheath $\times 80$ (b) Part of the same fibre at higher power. $\times 200$. (c) and (d) Arrowheads mark whole internodes showing signs of demyelination $\times 80$. ventral roots, cervical autonomic ganglia, and samples of peripheral nerve and muscle from the limbs were taken and processed using conventional neuropathological techniques.

Paraffin histology showed marked subperineurial oedema in all fascicles but no epineurial inflammatory infiltrates. Macrophages were present in the endoneurium.

Examination of semithin sections showed reduced numbers of myelinated fibres, the density of fibres having a normal appearance was $1,412 / \mathrm{mm}^{2}$. Most of these fibres were large (fig 1) and the histogram of fibre diameter distribution (fig 2) showed few small fibres but almost normal numbers of large fibres. It is possible that vesicular change in the myelin (visible by electron microscopy) accounts for the larger size of some of the fibres compared with age matched controls.

Degenerating myelin, often appearing as small clumps of granular material was seen frequently, but there was no clear evidence of demyelination at the light microscope level in the transverse sections.

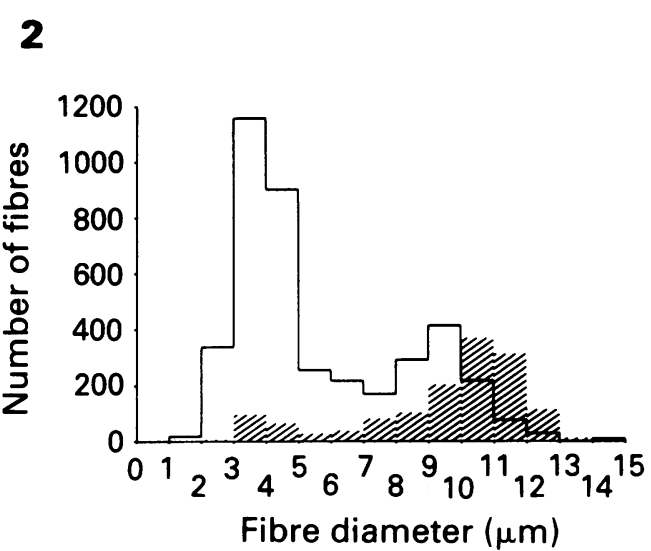

Figure 2 Histogram showing the distribution of total numbers of myelinated fibres in the patient (hatched) and in an age-matched control. There is selective loss of small fibres.

Teased fibres were most informative. The presence of myelin breakdown products could be identified along extensive lengths of many nerve fibres, particularly those of larger size. However, in many instances, an intact internode could be found along such fibres, indicating a process of demyelination rather than axonal degeneration. It was noted that the breakdown products of myelin were frequently small granular particles more typical of those seen in demyelinating conditions than the larger ovoids characteristic of early axonal degeneration. Figure 3 illustrates myelin changes affecting whole internodes which are flanked on either sides by internodes of a normal appearances. An early change seems to result in the paler and irregular staining myelin, compared with the appearance of adjacent normal internodes (fig $3 a$ and $b$ ); at this stage the sheath still seems to be intact. In other fibres the internodal myelin begins to show signs of disruption and discontinuities become apparent (fig 3c, d).

Electron microscopic examination showed
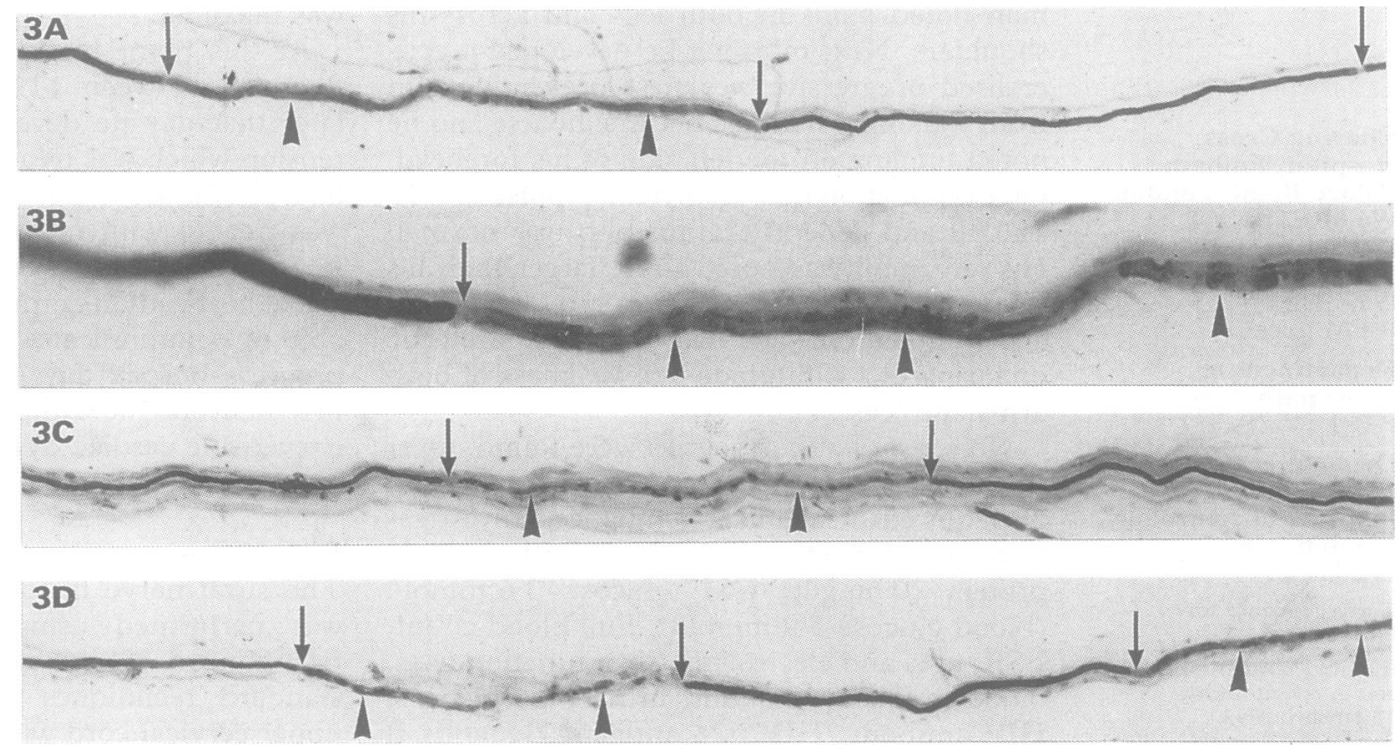
Figure 4 Electron micrograph showing a demyelinated fibre. The axon is small (maximum diameter about $1.5 \mu \mathrm{m}$ ) and appears shrunken with closely packed organelles. $\mathrm{Bar}=1 \mu \mathrm{m}$

Figure 5 Electron micrograph of a fibre with vesicular myelin; the axons is small and shrunken. Bar $=5 \mu \mathrm{m}$.

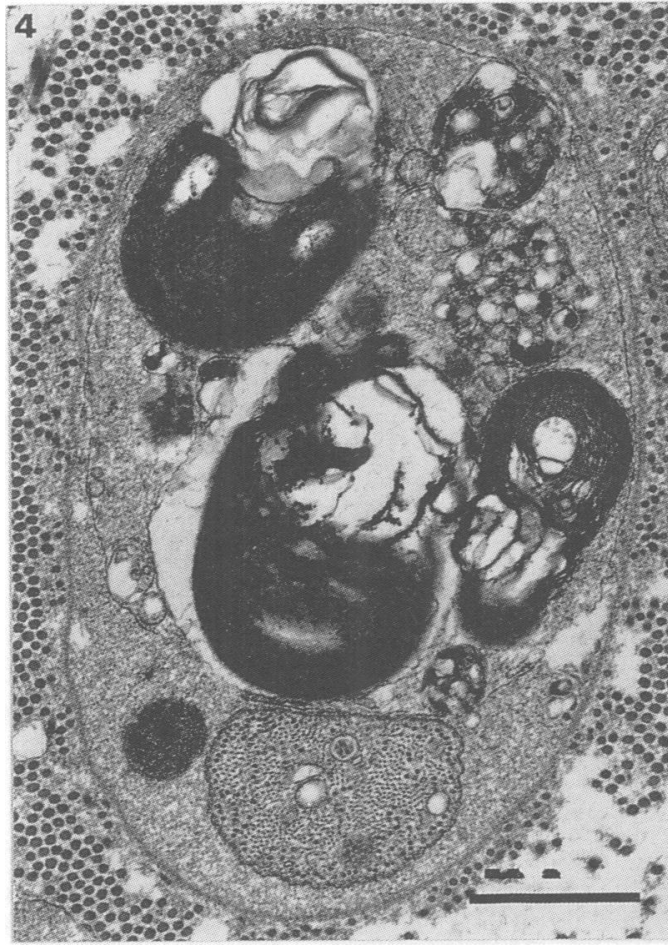

ated axons were seen and they were again of small size.

Loss of unmyelinated axons was indicated by the presence of stacks of Schwann cell processes, although the density of axons $\left(19,824 / \mathrm{mm}^{2}\right)$ was within normal limits for age. Some small demyelinated axons may have been mistakenly counted as unmyelinated axons. Occasional bands of Bunger were seen, evidence of earlier myelinated fibre degeneration. Macrophages and an occasional lymphocyte were identified, but only in modest numbers.

\section{Necropsy findings}

The brain was normal macroscopically, and histological examination of cortex, basal ganglia, cerebellum, brainstem (including oculomotor and Edinger-Westphal nuclei), olfactory and optic nerves and cervical spinal cord was normal. The eighth cranial nerve was not examined. The oculomotor nerves and lower cranial nerves, cervical dorsal and ventral roots, samples of median and sural nerves all had evidence of demyelination and Wallerian degeneration. Figures 6 and 7 illustrate regions in roots and median nerve, respectively, in which myelin loss is demonstrated, with adja-

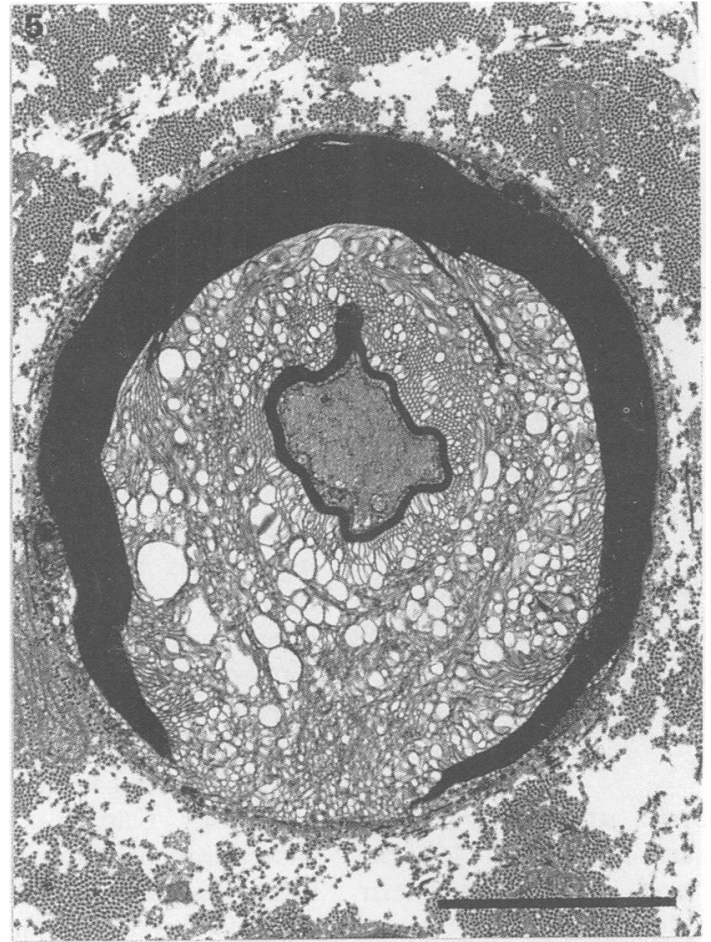
cent sections showing positive staining for axons. The cervical sympathetic ganglia revealed intact ganglion cells with evidence of demyelination and fibre degeneration in the preganglionic fibres. The vagus nerve in the neck also revealed widespread demyelination. Samples of sternomastoid, dorsal interossei, gastrocnemius and extensor digitorum brevis muscles all showed patchy focal muscle fibre atrophy and fibrosis, indicative of denervation and fascicles of intramuscular nerves showed demyelination.

\section{Discussion}

This patient had clinical evidence of a rapidly developing sensory, motor and autonomic neuropathy and fulfilled diagnostic criteria for GBS. ${ }^{6}$ Within 48 hours of his first symptom his pupils had become unreactive and pharmacological studies then confirmed them to have neither parasympathetic nor sympathetic innervation. ${ }^{7} \mathrm{He}$ also developed labile blood pressure followed by persistent hypertension and had evidence of sympathetic denervation of his skin. Sural nerve biopsy was consistent with a fulminating demyelinating neuropathy and postmortem found a severe sensorimotor and autonomic demyelinating neuropathy

numerous fibres containing myelin debris in which a demyelinated axon could be identified (fig 4); These axons were sometimes very small (1-2 microns) and would be difficult to recognise by light microscopy. In many fibres there was vesicular myelin (fig 5); it is suggested that this change might account for the pallor and irregularity of staining seen in affected internodes in teased fibres, and probably indicates incipient demyelination. Macrophages were occasionally seen near to the demyelinating fibres after careful searching, in only one instance was there any suggestion of myelin stripping. Some small completely demyelinoccurring in GBS syndrome ${ }^{89}$ and occurs in about $30 \%$ of patients. ${ }^{9}$ This usually manifests as sinus tachycardia, postural hypotension, reduced sinus arrhythmia, systolic hypertension, cardiac arrhythmias, which may be fatal, and both anhidrosis and excessive sweating. ${ }^{89}$ Pupillary abnormalities have rarely been described. The preganglionic sympathetic and parasympathetic fibres that supply the pupil are thinly myelinated. There have been four previous reports of pupillary involvement in 

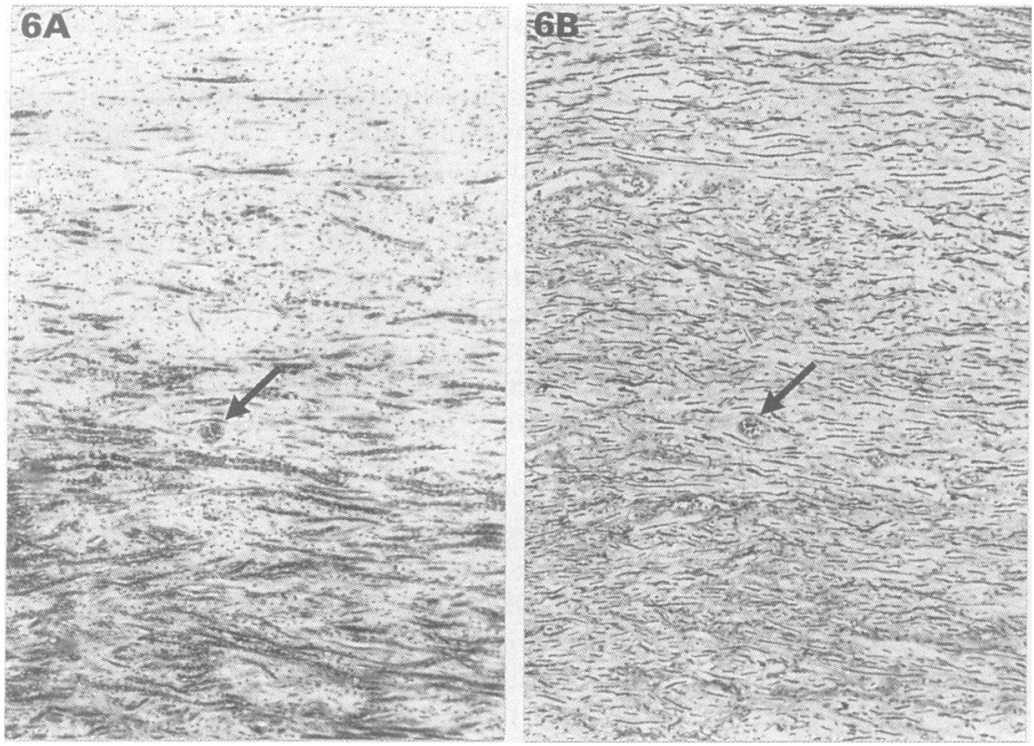

Figure 6 Longitudinal sections of spinal root. $\times 75$ (a) There is a region of myelin loss at the top of the picture (LFB/Nissl). (b) An adjacent section (arrows mark the same blood vessel) showing many axons throughout the whole root. Glees and Marsland.

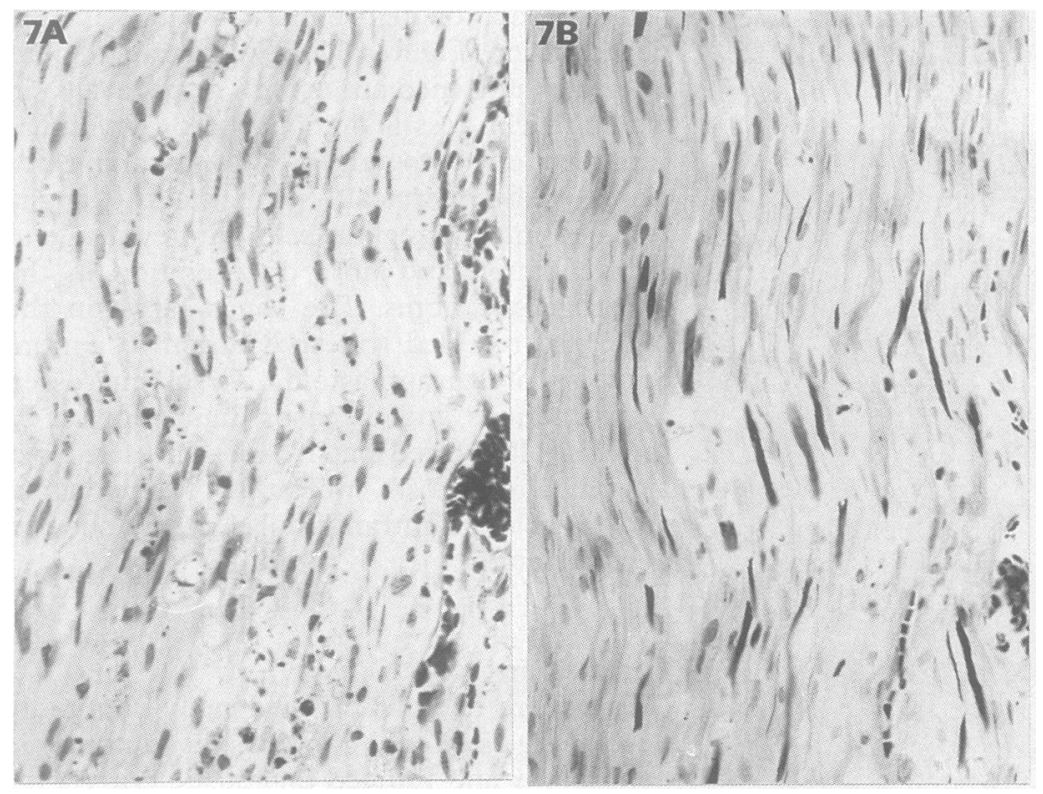

Figure 7 Longitudinal sections of median nerve. $\times 300$. (a) Myelin debris is seen but no intact myelin sheaths. (LFB/Nissl). (b) A nearby section shows positive staining for axons. Glees and Marsland. another was walking with a stick after 12 months ${ }^{10}$ and the third was breathing independently after 15 weeks ${ }^{12}$ and at 14 months was wheelchair bound. ${ }^{13}$ The fourth died ${ }^{11}$ and necropsy was performed (see below).

The nerve conduction studies in our case found no motor or sensory responses and one previous case had similar findings. ${ }^{4}$ One other case was studied earlier in the disease before significant paralysis and the only abnormality was reduced motor action potentials. ${ }^{11}$ Absent distal compound muscle action potentials (CMAP) occur in about $5 \%$ of patients with GBS. ${ }^{2}$ CMAPs below $20 \%$ of the lower limit of normal have been found to be prognostic of poor outcome. ${ }^{2}$

The clinical and electrophysiological features of our case are similar to the cases described as "an acute axonal form" of GBS. However, the pathological findings of the sural nerve biopsy, performed six days after the onset of neuropathic symptoms, indicate that the early pathological mechanism was demyelination. Necropsy examination of roots and peripheral nerves also show regions in which there is evidence of severe loss of myelin although axons were still present. Similar pathological findings of preserved axons with disrupted and degenerating myelin sheaths were reported by $\mathrm{Kanda}^{11}$ at necropsy of a patient who died eight days after the onset of neuropathic symptoms. These lesions were seen more frequently in nerve roots than in peripheral nerves, and were interpreted as evidence of primary demyelination. Interestingly, they also noted a relative preservation of large myelinated fibres. Another feature noted by Kanda was the relative paucity of inflammatory cells in regions where there was evidence of early demyelination. A relatively small number of inflammatory cells was also noted in the present case; macrophages were seen but not in numbers that might suggest they were involved in a florid cell-mediated demyelination. The inability to differentiate morphologically between macrophages whose function is to remove products of myelin breakdown and those involved in the immune-mediated destruction of myelin sheaths makes it difficult to decide what might be the mechanism of the pathology. Kanda et al $^{11}$ suggest that in their case demyelination is more likely to be due to humoral than cell-mediated factors, and this may also be the explanation in our case. Myelin vesicular change was seen frequently and appeared to indicate incipient demyelination.

The pathological findings in our case were similar to those reported in most patients in a necropsy series. ${ }^{3}$ That series included one case with a primarily axonal pathology without evidence of demyelination. This case was atypical. It first began at the age of six months, improved a little and then relapsed 14 months later and died 18 months after the original illness.

Without the use of teased nerve fibres and electron microscopy, the neuropathological changes in our case might have been mistaken for axonal degeneration. It seems possible that the case with necropsy described by Feasby et 
$a l^{4}$ as an axonal form of Guillain-Barré syndrome, a patient who died 28 days after onset of neuropathic symptoms and the case described by Honovar ${ }^{3}$ who died 18 months after onset (see above), might represent a later stage of a very severe demyelinating neuropathy as we have described.

1 Asbury AK, Arnason BG, Adams RD. The inflammatory lesion in idiopathic polyneuritis: its role in pathogenesis. lesion in idiopathic polyneurit

2 Cornblath CR, Mellits ED, Griffin JW, et al. Motor conduction studies in Guillain-Barré syndrome: description and prognostic value. Ann Neurol 1988;23:354-9.

3 Honavar M, Tharakan JKJ, Hughes RAC, Leibowitz S, Winer JB. A clinicopathological study of the GuillainBarré syndrome. Nine case and literature review. Brain 1991;114:1245-69.

4 Feasby TE, Gilbert IJ, Brown WF, et al. An acute axonal form of Guillain-Barré polyneuropathy. Brain 1986;109:1115-26.

5 Jacobs JM, Love S. Qualitative and quantitative morphology of human sural nerve at different ages. Brain 1985;108:897-924

6 Arnason BGW. Acute inflammatory demyelinating polyradioculoneuropathies. In: Dyck PJ, Thomas PK, Lambert EH, Bunge eds. Peripheral neuropathy. Philadelphia: W B Saunders, 1984:1811-25.

7 McLeod JG, Turk RR. Disorders of the autonomic nervous system. PII. Investigation and treatment. Ann Neurol 1987;21:519-29.

8 Turk RR, McLeod JG. Autonomic dysfunction in GuillainBarré syndrome. I Neurol Neurosurg Psychiatry 1981; 44:983-90.

9 Winer JB, Hughes RAC. Identification of patients at risk of arrhythmia in the Guillain-Barré syndrome. Quart $\mathcal{f}$ Med arrhythmia in the

10 Carroll WM, Mastalgia FL. "Locked-in coma" in postinfective polyneuropathy. Arch Neurol 1979;36:46-7.

11 Kanda T, Hayashi H, Tanabe H, Tsubaki T, Oda M. A fulminant case of Guillain-Barré syndrome: topographic and fibre size related analysis of demyelinating changes. $\mathcal{f}$ Neurol Neurosurg Psychiatry 1989;52:857-64.

12 Coad NR, Byrne AJ. Guillain-Barré syndrome mimicking brainstem death. Anaesthesia 1990;45:456-7.

13 Hassan T, Mumford C. Guillain-Barré syndrome mistaken for brain stem death. Postgrad Med f 1991;67:280-1.

14 Hawkes CH, Bryan-Smith I. The electroencephalogram in the "Locked-in" syndrome. Neurology 1974:24: 1015-8.
George Sumner Huntington (1850-1916) was born in East Hampton, Long Island and trained with his father Dr George Lee Huntington in local practice before attending the College of Physicians and Surgeons at Columbia University, graduating in 1871. A year later he moved to Pomeroy, Ohio and on February 151872 read his paper on chorea ${ }^{1}$ before the Meigs and Mason Academy of Medicine in Middleport, Ohio. He was twenty two years old.

"It is as common and is indeed, I believe, more common among men than women, while I am not aware that season or complexion has any influence in the matter. There are three marked peculiarities in this disease:

1. Its hereditary nature.

2. A tendency to insanity and suicide.

3. Its manifesting itself as a grave disease only in adult life."

Addressing the New York neurological society in 1909 he recalled:

"... riding with my father on his rounds, I saw my first case of 'that disorder'... the way the natives always referred to the dreaded disease... We suddenly came upon two women, mother and daughter, both tall, thin, almost cadaverous, both bowing, twisting, grimacing. I stared in wonderment, almost fear."

The affected family was further studied (by Jelliffe, Tilney and by Vessie ${ }^{2}$ ) and traced to Bure in Essex, whence they sailed to Boston Bay in 1630. Over a thousand descendants are thought to have succumbed, many being tried for witchcraft since their chorea was regarded as "a derisive pantomime of the sufferings of the Saviour during crucifixion."

Huntington spent the rest of his life in Dutchess County in general practice. He was a keen sportsman, artist, and flute player who modestly referred to his work as "my virgin contribution to medical lore." JMS PEARCE

1 Huntington G. On chorea medical and surgical reporter, Philadelphia 1872;26:317-21

2 Vessie [J Nerv Ment Dis 1932;76:553-73.]. cited by RN DeJong. In: Haymaker's Founders of Neurology, 2nd ed $1970 ; 452-5$. 